\title{
GENERAL PROPERTIES OF THE TWO GROUPS OF GLOBULAR CLUSTERS AND THEIR VARIABLE STARS
}

\author{
A. V. MIRONOV and N. N. SAMUS'
}

Sternberg Astronomical Institute, Moscow, U.S.S.R.

\begin{abstract}
The dependences of the numbers of variable stars in globular clusters on the chemical composition are studied. For given metallicity the numbers of $R R$ Lyrae stars reduced to some definite total number of stars in the cluster are different for the two groups of globular clusters introduced by Mironov.
\end{abstract}

Recently Mironov (1973a, b) suggested the separation of the globular clusters of our Galaxy into two groups on the basis of the morphological characteristics of their colour-magnitude diagrams. Originally this separation was dependent on a somewhat unnatural parameter $I M \times Y$. Later it became possible to undertake such a separation without taking into account the parameter $I M \times Y$ and to propose a physical explanation for the separation (Mironov and Samus', 1974). The main properties of the two above-mentioned groups of globular clusters are presented in Table I. One should remember that the majority of the quantities in Table I were determined using methods which are not free from systematic errors, so that these quantities must be considered only as relative ones.

\section{TABLE I}

\section{Group I}

(1) Typical representative: M 13

(2) $-1.3 \geqslant[m / H] \geqslant-2.1$

(3) There is no dependence of $B /(B+R)$ on $Y$

(4) No relation between $[m / H]$ and $Y$

(5) $0.22 \leqslant Y \leqslant 0.33$

(6) No relation between the distance from the galactic centre $D$ and either $[m / H]$ or $Y$

(7) $B /(B+R)=0.90 \pm 0.05$

(8) $t \sim 25 \times 10^{9}$

\section{Group II}

Typical representatives:

M 92, M 3, 47 Tuc

$-0.02 \geqslant[m / H] \geqslant-2.17$

The more $Y$, the less $B /(B+R)$

The more $[m / H]$, the more $Y$

$0.23 \leqslant Y \leqslant 0.42$

Both $[m / \mathrm{H}]$ and $Y$ increase when $D$ decreases

$0 \lesssim B /(B+R) \lesssim 1$

$t \sim 12 \times 10^{9}$

\section{Comments}

(2) See Kukarkin (1974).

(3)-(7) For $B /(B+R)$ see Mironov (1973a), for $Y$ see Mironov and Samus' (1974).

(8) $t$ was calculated using Sandage's (1970) formula. 
Naturally the reduced frequency of RR Lyrae stars in a globular cluster (i.e. the number of variables reduced to some definite total number of stars in the cluster) is determined by the number of stars in the RR Lyrae gap, so there must exist a very tight relation between the reduced frequency and the value $B /(B+R)$. Since $B /(B+R)$ for given $[m / H]$ is larger for Group I clusters than for Group II clusters, the number of variables in the clusters of Group I is expected to differ from the number of variables in the clusters of Group II for given metallicity. On average that is the case in reality (see Figure 1).

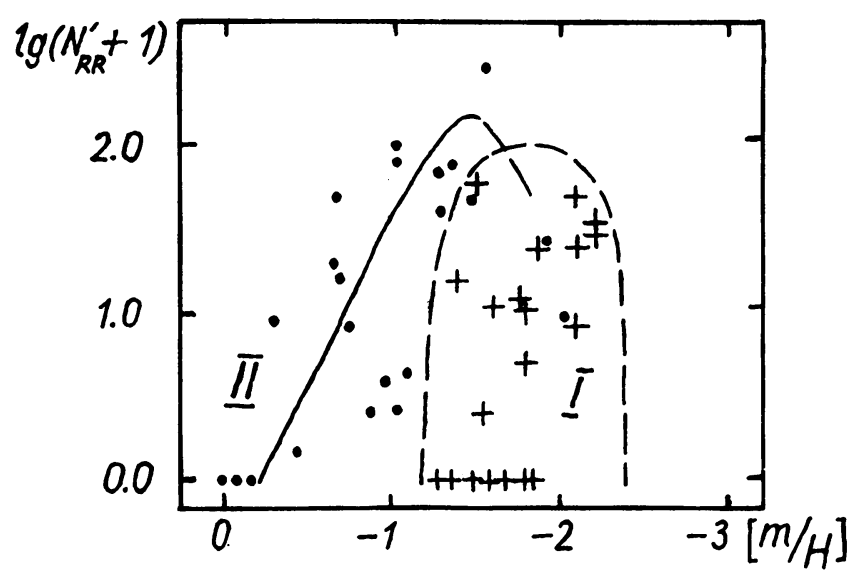

Fig. 1. The reduced numbers of RR Lyrae variables in globular clusters of different metallicity.

The limited sample of W Vir and RV Tau stars in globular clusters did not allow us to find any significant difference between the character of the dependence of reduced numbers of variables on the chemical abundance for the two groups. The mean relations are shown in Figure 2.

\section{Explanation of some definitions}

$B \quad=$ number of stars on the horizontal branch on the blue side of the RR Lyrae gap;

$R \quad=$ same on the red side of the gap;

$Y \quad=$ helium abundance;

$[m / \mathbf{H}]=\log (m / \mathbf{H})-\log (m / \mathbf{H})_{\odot}=$ metal to hydrogen ratio with respect to the solar value;

$I M=$ metallicity index. This quantity corresponds more or less to $[\mathrm{m} / \mathrm{H}]$. For the relation between $I M$ and $[m / H]$ see Kukarkin (1973). 

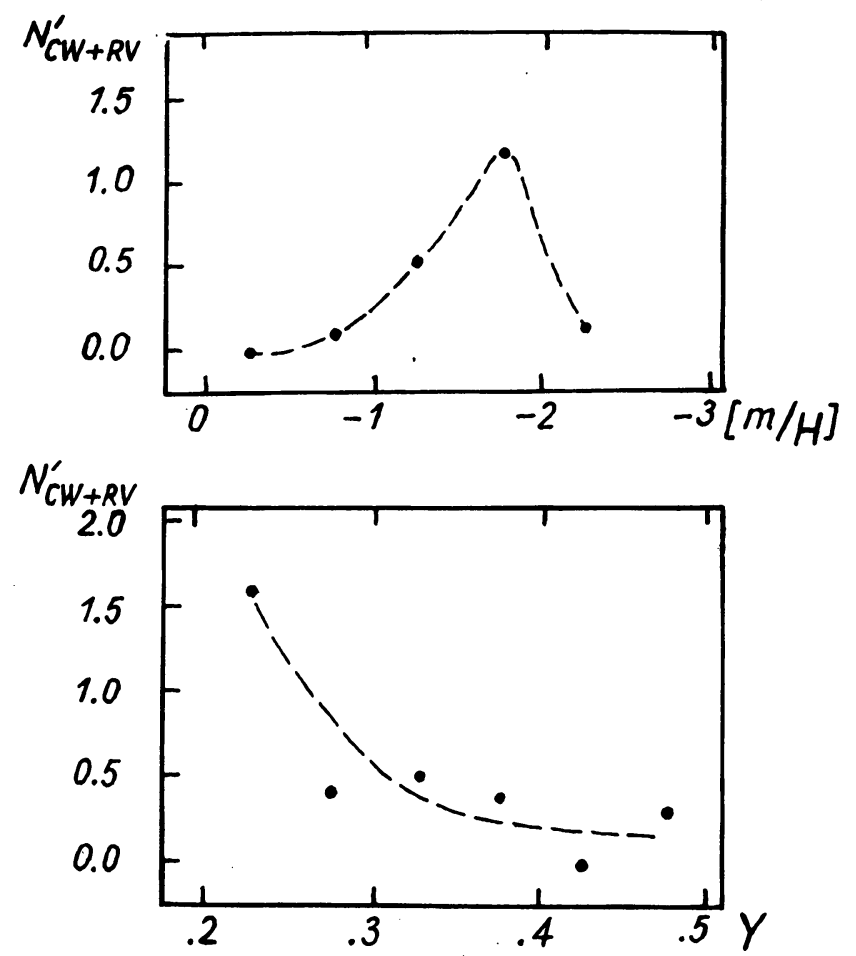

Fig. 2. The dependences of the reduced numbers of $\mathrm{W}$ Vir- and RV Tau-type variables on the chemical composition parameters $[m / \mathrm{H}]$ and $Y$.

\section{References}

Kukarkin, B. V.: 1973, Astron. Tsirk. U.S.S.R., No. 780.

Kukarkin, B. V.: 1974, Globular Star Clusters, "Nauka" Publishing House, Moscow.

Kukarkin, B. V. and Russev, R. M.: 1972, Astron. Zh. 49, 121 = Soviet Astron. $16,95$.

Mironov, A. V.: 1973a, Astron. Zh. 50, 27.

Mironov, A. V.: 1973b, in J.D. Fernie (ed.), Variable Stars in Globular Clusters and in Related Systems, D. Reidel Publ. Co., Dordrecht, p. 229.

Mironov, A. V. and Samus', N. N.: 1974, Variable Stars 19, 337.

Sandage, A. R.: 1970, Astrophys. J. 162, 841. 